\title{
Adoption of Electronic Health Records
}

\section{A Qualitative Study of Academic and Private Physicians and Health Administrators}

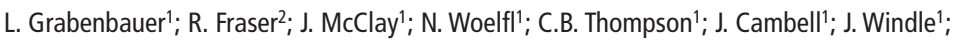

${ }^{1}$ University of Nebraska Medical Center; ${ }^{2}$ University of Missouri

\section{Keywords}

Adoption, health information technology, workflow

\section{Summary}

Objective: Less than $20 \%$ of hospitals in the US have an electronic health record (EHR). In this qualitative study, we examine the perspectives of both academic and private physicians and administrators as stakeholders, and their alignment, to explore their perspectives on the use of technology in the clinical environment.

Methods: Focus groups were conducted with 74 participants who were asked a series of openended questions. Grounded theory was used to analyze the transcribed data and build convergent themes. The relevance and importance of themes was constructed by examining frequency, convergence, and intensity. A model was proposed that represents the interactions between themes. Results: Six major themes emerged, which include the impact of EHR systems on workflow, patient care, communication, research/outcomes/billing, education/learning, and institutional culture. Academic and private physicians were confident of the future benefits of EHR systems, yet cautious about the current implementations of EHR, and its impact on interactions with other members of the healthcare team and with patients, and the amount of time necessary to use EHR's. Private physicians differed on education and were uneasy about the steep learning curve necessary for use of new systems. In contrast to physicians, university and hospital administrators are optimistic, and value the availability of data for use in reporting.

Conclusion: The results of our study indicate that both private and academic physicians concur on the need for features that maintain and enhance the relationship with the patient and the healthcare team. Resistance to adoption is related to insufficient functionality and its potential negative impact on patient care. Integration of data collection into clinical workflows must consider the unexpected costs of data acquisition.

\section{Correspondence to:}

Lisa Grabenbauer, MS, MBA

982265 Nebraska Medical Center

Omaha, Nebraska 68198-2265, USA

E-mail: Igrabenbauer@unmc.edu

\author{
Appl Cin Inf 2011; 2: 165-176 \\ doi:10.4338/ACI-2011-01-RA-0003 \\ received: January, 122011 \\ accepted: April 4, 2011 \\ published: Mai 25, 2011
}

Citation: L. Grabenbauer, Fraser R, McClay J, Woelfl N, Thompson CB, Cambell J, WindleJ. Adoption of EHR aqualitative study of academic and private physicians and health administrators. Appl Clin Inf 2011; 2: 165-176

http://dx.doi.org/10.4338/ACl-2011-01-RA-0003 


\section{Introduction}

Despite the potential advantages of the Electronic Health Record (EHR) [1-5], adoption of technology has been slower in health care than in other sectors of industry. Currently, the use of an EHR in ambulatory settings ranges from $42-90 \%$ in the United Kingdom, Western Europe, and Eurasia, with North American usage at less than 30\%. However, within hospitals, adoption rates among these same nations are less than $10 \%$. A comprehensive EHR, linking inpatient and outpatient data, exists in less than $20 \%$ of hospitals in the United States [6]. Efforts to stimulate the active pursuit of Health Information Technology (HIT) were supported by President George W. Bush, and with increased vigor by the current Obama administration [7, 8].

The American Recovery and Reinvestment Act, signed by President Obama in 2009 to provide economic stimulus, encourages the development of HIT systems that provide "meaningful use". Criteria are defined in the Health Information Technology for Economic and Clinical Health (HITECH) Act and include quality, safety, and efficiency improvements. Adoption of an EHR that satisfies these criteria will be rewarded by financial incentives [9]. This initiative will require institutional transformations in culture regarding adoption of technology and the management of change.

Physician resistance has often been cited for this delay [10]. Academic physicians are expected to be less likely to resist adoption than private physicians because they are less impacted by the cost of technology and the work of data entry. Private physicians bear the cost of hardware, software and maintenance, interfaces and education for their private practices [11]. In addition, they must share information between disparate practices. Therefore, we speculate that a difference in perceptions toward EHR systems may exist between academic and private physicians, which could best be examined in an institution that involves both groups. We also included administrators, who are often involved in decisions on technology purchases.

Rogers has provided foundational work to address the problem of adoption of technology in various domains[12]. Moore further expands the discussion by describing the chasm between the initial proponents of a technology (the innovators and the early adopters), and the early majority - the group that succeeds in igniting the momentum of adoption [13]. This model is often used to describe EHR adoption, with physicians being the point of resistance.

Lorenzi presents strategies for overcoming adoption barriers which span organizational and domain boundaries and identifies categories of issues which include design, management, organization, and assessment. The successful adoption requires an understanding of EHR users and their work setting [14-17].

Our study was conducted at The Nebraska Medical Center, an independent, not-for-profit, 689 bed private hospital with 412 academic physicians and 581 private physicians. This unique institutional culture allows us to investigate whether potential causes for the low adoption rate are due to the differences between academic and private physicians. Institutional culture is also shaped by the decisions of administrators, who expect that the integration of an EHR into medical practice will lead to benefits including increased patient safety and prevention of medical errors [18-21]. The consideration of both physicians and administrators as direct stakeholders, and their alignment, is necessary to explore the keys to successful adoption and use of technology.

\section{Methods}

This research was part of an Integrated Advanced Information Management Systems (IAIMS) supported study. The research objective was to explore how private and academic physicians differ in their perception and adoption of technology within the hospital setting. More specifically, the aims are (1) to document EHR interactions that impact adoption, (2) compare these characteristics between the physician groups, (3) determine how administrators determine EHR value, and (4) to compare the views of physicians and administrators.

A qualitative approach was used to collect and analyze data using grounded theory $[22,23]$. This allows better understanding of the social phenomena related to physician perceptions leading to the adoption of technology. This approach allows for collection of a rich contextual narrative that provides meaningful insight into the potential variables that impact on behavior ( Fig .1). 


\section{Participant Profile}

A convenience sample of academic and private physician and administrators was obtained based on recommendations of the study's steering committee. The steering committee was assembled to oversee the IAIMS grant and represents thought leaders from across the institution. Selection of the sample was based on users who were considered thought leaders, and representative of early majority adopters, who are more pragmatic in their emphasis on solutions rather than on technology.

The study sample of 74 was divided into four stakeholder categories and included 38 academic practitioners (AP), 14 private practitioners (PP), 12 university administrators (UA) and 10 hospital administrators (HA).

\section{Focus Group Design}

Twenty-four sessions were conducted with physicians and administrators associated with either the University of Nebraska Medical Center (academic practice) or its affiliated private hospital, The Nebraska Medical Center (private practice). All physicians used the same comprehensive EHR (GE CareCast 5.1.7) while caring for patients at TNMC. Most physicians included in the study, whether academic or private, work in different healthcare systems that use different EHR's.

Participants were asked open-ended questions related to their use and expectations for an EHR system. Questions related to their perceptions and attitudes regarding patient care, physician workflow, care team interactions, flow of health information, outcomes and clinical research, and the provider's ability to learn. Interviews and analysis took place between August, 2006 and March, 2007. An average of 7 individuals participated in each focus group which lasted 1 to 3 hours.

Group proceedings were audio-recorded and transcribed to allow coding and analysis using NVivo v7.0 software. Theoretical sampling continued until saturation was reached, and no more new ideas surfaced during the discussions [24]. At that point sub-group comparisons were performed. Two investigators independently and systematically reviewed transcripts to identify themes and sub-themes unique and similar across all groups. Themes were verified by a third investigator. Systematic coding scales included frequency (the number of times that the topic appears in the analysis), convergence (whether the topic extends across subject classification groups), and intensity (the emotion and importance of topic to the speaker) of the data elements. Using an iterative process, these themes were revised until a consensus was achieved among all three investigators. The first phase of 18 focus groups did not yield saturation, so an additional 6 sessions were added until saturation of responses was reached.

\section{Results}

The systematic review of the transcripts revealed six major themes, which include the impact of health information technology on:

- Workflow - the physical interaction of the healthcare provider with information and with patients, which includes the amount of time needed to capture, retrieve and process information.

- Patient Care - the focus of effort centering on the relationship between the provider and the patient.

- Communication - the interaction between the members of the healthcare provider team, and the methods needed to facilitate the exchange of information.

- Research/Outcomes/Billing - the use of data in a structured and summarized way to satisfy research, outcomes and billing, including capture of data in the appropriate formats.

- Education/Learning - the use of an EHR to support the provider's medical education, as well as any learning that is required to effectively use the EHR system.

- Culture - the issues related to how an EHR affects culture and the underlying beliefs and attitudes of the different groups of participants regarding use of the technology.

The significance of these themes is explained by the frequency of comments contained in $>$ Table 1 . Themes are ranked by the number of comments coded to that theme. Although comments related to and private physicians and health administrators 
institutional culture were most frequent, it was listed at the bottom to indicate its pervasiveness in respondent comments.

Further analysis resulted in the cross-case analysis documented in $>$ Table 1, which shows similarities between academic and private physicians and between university and hospital administrators. This is sharply contrasted by the differences between physicians and administrators. This indicates strong convergence between academic and private physicians on four of six themes, responding very negatively to physician workflow, patient care, communication and culture. Physician groups differed on the impact of education and learning, with academics slightly positive, and private physicians slightly negative, and their view of outcomes and research, where academic physicians were slightly positive and private physicians neutral. University (academic) and hospital (private) administrators also exhibited convergence across five of six themes, responding with neutral to positive comments, differing only on the theme of education and learning. In sharp contrast, physicians and administrators differed on nearly all themes, with the least divergent themes of outcomes and research and education and learning. Detailed descriptions follow.

\section{Physician Workflow}

Both academic and private physician groups contributed frequent comments on the theme of physician workflow, indicating convergence in this area between the physician groups. The physicians concurred with negative perceptions on workflow. They cited loss of efficiency produced by the use of technology - more time needed for data entry, less time spent with patient interaction, frequent logins, and tedious standardized forms that compromised the richness of language and depersonalized the relationship with the patient. Their emotional use of language and tone of voice indicated the intensity of their reaction.

"If you listen to how we describe, at least for us, the way that we do our life, this is gonna be a lot more work, uh, to do it this way and for the benefit of the organization." (AP)

"So instead of being able to get all the information on the computer or all the information from the chart, you have to go to two sources to get the information that I used to be able to look at a sheet [of paper] for 20 seconds and there's just not enough access in the locations that we need to get the information." (AP)

"I wrote down all of my orders just like I did, got a little crib sheet and spent time going through it. It wasn't faster... I'm still doing it, but it's, ought to be able to slide through and just grab what I need and go, "Click, click, click, click". (PP)

In contrast, administrators made fewer comments regarding workflow, and were positive about the potential benefits of the collection of data by physicians, with few comments indicating the negative impact to workflow as articulated by physicians.

"I see, there's lots of different products out here but there's a big distinction in my mind of electronic health record and what we're trying to build here is the electronic medical record which is you know, captures a lot of the information but also is useful in the, you know, care setting and delivering that care." (HA)

\section{Patient Care}

Comments on the impact of information technology on patient care occurred with similar frequency across all four groups, identifying convergence of this important theme to both physician and administrator groups. However physicians responded intensely and often negatively, citing gaps between EHR system features and physician needs.

"... every single thing is in there electronically, you have to wade through just a ton of administrative $c^{* *} p$ and follow on quality reports from physical therapy you know, you're just trying to find what the ID docs [recommend] that you should do for the antibiotics—" (AP)

"Whatever it would take to get that to be the standard of care but as every physician walking into a patient encounter walks in with electronic health record would be a huge improvement in care." (AP) 
"And so the quality improvement issues are really, I mean right now we're focusing in on precision, we have a lot of people from outside the industry who don't understand they're dealing with biological organisms so they come in and want to affect us with manufacturing thoughts." (AP)

"I've had patients come to me and ask for referrals to other physicians because they feel that all he does is to look at the computer now when they're in there." (PP)

Administrators were neutral to mildly positive. They were optimistic about improved outcomes for better patient care, trends for patient ownership of health data, the movement toward patient-centric care, and the need to systematically support the continuity of care.

"One of the outcomes in going forward will be that we will be able to measure some things, improvement with care that today we can't because there is really no way to measure it." (HA)

"I don't think we have the knack yet of realizing that the patient is still the center of focus and a lot of people spend too much time away at the electronic medical record." (HA)

\section{Communication}

Care team interactions were cited frequently by all groups, but were perceived negatively for both physician groups who noted the reduced effectiveness of communication - unclear transfer of patient responsibility, and fewer checks and balances, while administrators supported a more neutral view.

"We should force the technology to work with us. And if our intention is to have team-based rounds with nurses and physicians and other providers all communicating among one another, then we need to be sure that happens." (AP)

"... perhaps the lesson we can learn from the VA is don't build a lot of closets and stick computers in them where people could go inside and shut the door and hide." (AP)

"I think there is potential for uh, big problems with the physicians not interacting enough with nurses. ... And I think there is risk here for uh, sterilizing or dividing the relationship between the physician and the nurses." (PP)

\section{Research and Outcomes}

The impact of HIT on research and outcomes was more frequently commented on by academic physicians, who noted the potential to improve outcomes and research by overcoming cumbersome data entry and standardization of redundant data elements.

"Show me that it helps me care for patients better. I mean, even if it takes more time, I'll do it if it helps me care for my patients better." (AP)

University and hospital administrators were neutral to positive in their responses. However, their comments were focused on the potential of improved compliance and billing, better control of costs and data for decision-making, rather than established benefits. They also cited needed improvements of data interfaces and data collection.

\section{Education and Learning}

The topic of learning was interpreted very differently by the two groups of physicians studied. Academic physicians viewed the question from a teacher's perspective - as medical education. Private physicians viewed it from a learner's perspective-learning about how to use the technology. Not surprisingly, education was most frequently commented upon by academic physicians who were optimistic about improved learning at the point-of-care, supporting the ongoing educational needs of physicians. However, the academic physicians were also cautious about relying too much on technology. 
"Technology is not a substitute for the creativity required for the art of medicine ... fuzzy logic, complex thinking". (AP)

"The value of learning through experience can't be overridden by technology". (AP)

Private physicians made negative comments on learning, but referred specifically to system training, citing the steep learning curve and long hours required prior to use of new EHR applications.

\section{Culture}

A significant discrepancy exists in the perception of EHR impact on the institutional culture between physician and administrators. There were frequent comments by each group, but the intensity was different-academic physicians were negative, private physicians even more so - citing limited vision for EHR's and insufficient support for current EHR projects, unclear data ownership, the existence of data silos, hierarchical decision making and the influence of external agencies and mandates, and fear that the data would be used against them.

University administrators were weakly positive, while the hospital administrators were consistently positive about improved throughput linking laboratory and diagnostics, improved compliance and billing, better control of costs, improved outcomes, the trend for patient ownership of data, the movement toward patient-centric care, the influence of national trends and national initiatives aimed at improving patient care and safety. However, they also realized the current problems caused by the proliferation of data silos.

"Well I think doctors are accustomed to having things shoved down their throat. By ... the government, the hospitals, third party payers..." (PP)

"But I would hope that it would be physician-driven, a physician effort and then people could buy into it and think it was a good idea for physicians and for patient care. And it wasn't shoved down anybody's throat..." (AP)

"We have a jigsaw puzzle... And we know in the end what the picture should look like, but we can't put the pieces together." (HA)

\section{Discussion}

The six themes that were identified were further defined by their relationships. Surprisingly, we found that academic and private physicians have a high level of agreement (frequency, intensity and convergence) on the triad of patient care, workflow and communication. They both expressed concerns about the creation of data, which is reflected in these themes. Their reactions where mixed on the topics of outcomes and research and education and learning.

"It's like Christians, you could be Methodist, you could be Lutheran, whatever. And 95\% of doctors are the same, it's the other $5 \%$ we're gonna fight over." (PP)

The relationship of physician workflow and communication on patient care was strongly articulated by physicians, while outcomes and research and education and learning were also perceived as impacting on patient care. These themes are shown in $>$ Figure 2 . The overlap of culture with other themes indicates the pervasiveness and impact of institutional culture.

A number of important studies by Ash and others [25-31] identify previously overlooked components to lagging adoption - the unanticipated consequences of deploying EHR systems with limited design input from providers. Unintended consequences can be grouped into two categories. The first category, consequences related to entering and retrieving information, include system interfaces that don't tolerate workflow interruptions, allow orders to be entered for the wrong patient, or require complex structured data entry between multiple screens. The second group, those consequences associated with communication and coordination, include breakdowns in the management of responsibilities and tasks related to validation of treatment and transfer of patient responsibility, and the emergence of workarounds. The study base for much of this research looks at custom-developed systems that reside within the structure of the institution. These systems may proand private physicians and health administrators 
vide satisfied users, but this approach limits the availability of such costly solutions to large, funded academic institutions [32, 33].

Issues of misaligned incentives, slow standards adoption, and the identification of essential product features were identified by Middleton as early as 2004 [34, 35]. Our study indicates that, despite our presumption of differences, both academic and private physicians believe in the potential benefits of an EHR system, yet differ in how they articulate the costs and benefits. Discussions of cost centered on time needed to learn and use features that changed workflow and limited time with the patient. The results of our study indicate that both private and academic physicians were surprisingly similar in their need for features that maintain and enhance the relationship with the patient.

In summary, academic and private physicians both express serious concerns about the impact of an EHR on patient care, physician workflow, team communications, and culture. Academic physicians were optimistic about HIT data for outcomes and research and education and learning. Private physicians were less compelled by outcomes and research. In addition, they expressed concern about the effort required to learn and adapt to new systems. Physicians were joined in their belief that technology was being forced upon them and they are expected to adapt to technology. Administrators also showed a high level of similarity between university and hospital, similarly positive on workflow, patient care, communication, outcomes and research and their neutrality to culture. University administrators were optimistic about EHR impact on education and learning. Administrators simply believed that adoption of an EHR is necessary and will improve patient care.

An important divergence between groups may be explained by the difference between the creation of data and the use of data. The administrators' view is that the organizational use of data justifies the creation of data. They believe that creation of administrative data is the primary job of the EHR, and eagerly anticipate the availability of the data for quality and outcome measurements. In contrast, the physician's view is that data creation drives data usage. They feel that technology has been pushed on them at the expense of their efficiency, teamwork, and their time spent with the patient. Further, they are concerned that data creation drives a desire by administrators for greater data usage. Physicians believe that EHR's are inevitable, but desire a system that facilitates, not hinders, their ability to manage patients. Today, private and academic physicians believe that EHR is a solution for administrators, and that the benefits of better EHR data are far beyond their reach.

\section{Study Limitations}

This study was performed at a single medical center with a single EHR, which may limit generalization. However, nearly all physicians included in the study used different EHR's at different health systems and no differences in responses was detected. Years of experience has been proposed as a potential modifier [36], but we did not record the subjects' age or years of experience in this study. While relationships between themes were identified, more investigation is needed to clearly define the causal relationships between physician workflow, communication, and patient care. Culture was defined as a theme that provided an underlying foundation for other themes, but the nature of that relationship also requires more study, and may identify the values that underlie the responses and reactions of the stakeholders.

The view of physician adoption as a complex social phenomenon allows for discovery of the experiences, beliefs and values that hinder or encourage adoption. The resulting model provides a foundation for further study that includes the rich description of a technical system that is inseparable from its surrounding cultural system. Continued research is needed to better understand and resolve the trade-offs among competing values among the multiple stakeholders, which include both physicians and administrators.

\section{Conclusion}

An aggressive ten-year goal of universal EHR adoption by 2014 was set by President Bush. Projections imply that this goal is unlikely without incentives and product innovations[37]. A 2010 report from the President's Council of Advisors on Science and Technology outlines financial incentives to 
encourage progress in the areas of information exchange that benefits patients as consumers, clinicians and researchers [38].

We contend that physician adoption of EHR systems will be driven by how well EHR's support physician workflow, communication and patient care. This is not solved by financial incentives. Rather, it is a more complex resolution of the balance of tension between adequate design and increasing requirements for data use. Specifically, the solution will include improving the usability of systems for data entry, integrating into workflow and enhancing communication. This shift drives the effort beyond remedies for physician resistance to an intense focus on the design of elegant systems that match physician requirements and exceed the limits of current expectations. Success is dependent on technology that is designed to fit the needs of physician, with a unifying goal to improve patient care.

\section{Clinical Relevance Statement}

Our study demonstrates that resistance to adoption is related to insufficient functionality and its potential negative impact on patient care. Integration of data collection into clinical workflows must consider the unexpected costs of data acquisition. This study will help aid in the design and implementation of future clinical health information technology, and outlines the different concerns of stakeholders which include both private and academic practitioners and administrators.

\section{Conflicts of Interest}

The authors declare that they have no conflicts of interest in the research.

\section{Human Subjects Protections}

The study was performed in compliance with the World Medical Association Declaration of Helsinki on Ethical Principles for Medical Research Involving Human Subjects, and was reviewed by the University of Nebraska Medical Center Institutional Review Board. Informed consent was obtained in all cases.

\section{Acknowledgments}

This project was funded by a grant from the National Library of Medicine, grant number 5 G08 LM008271-02, titled "Advancing Academic and Community Practices through IAIMS." 


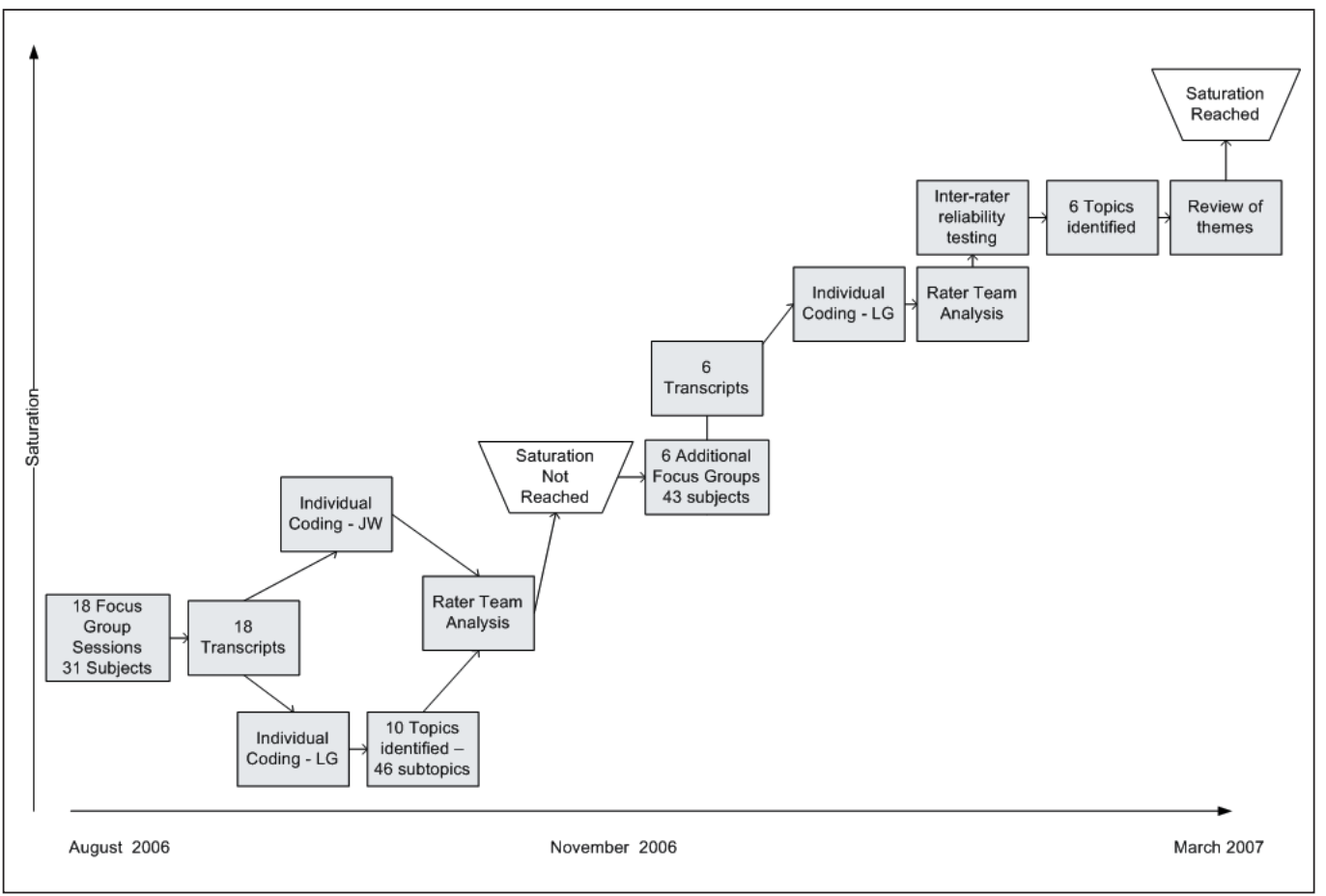

Fig. 1 Research timeline and methodology.

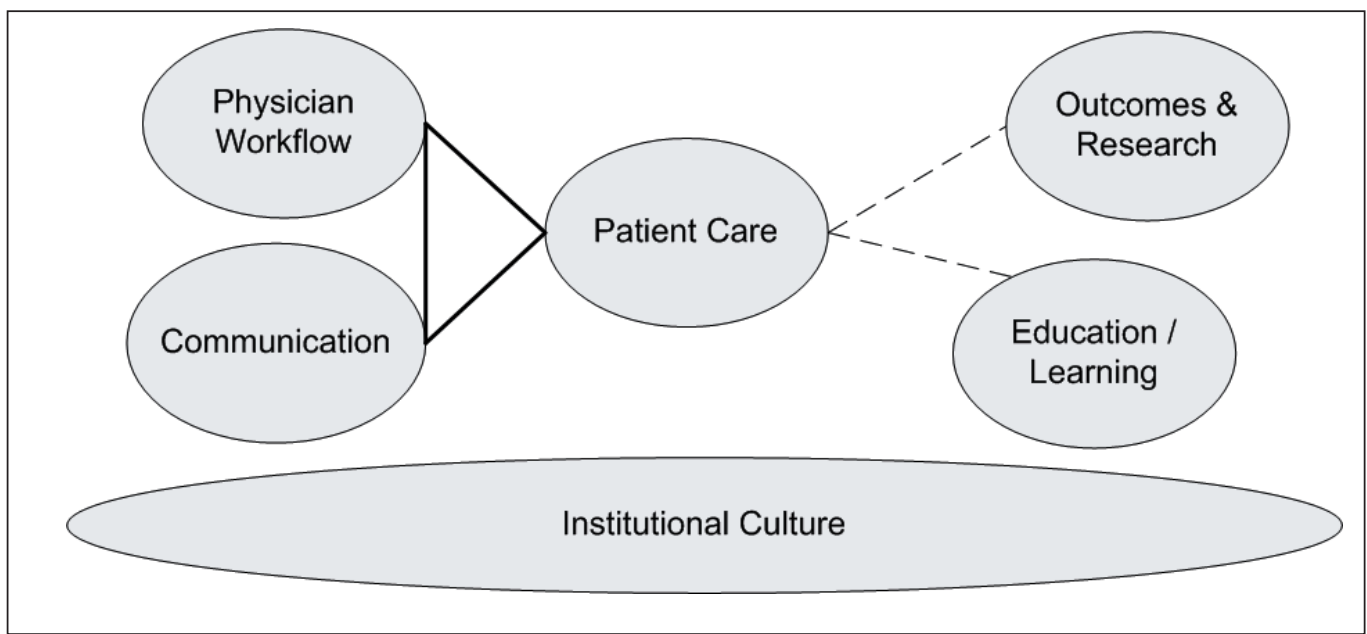

Fig. 2 Six adoption themes defined. 
Table 1 Frequency of themes emerging from focus group responses

\begin{tabular}{|c|c|c|c|c|c|}
\hline \multirow[t]{2}{*}{ Themes } & \multicolumn{5}{|c|}{ Subject Groupings } \\
\hline & $\begin{array}{l}\text { Academic } \\
\text { Physicians } \\
\text { (AP) }\end{array}$ & $\begin{array}{l}\text { Private } \\
\text { Physicians } \\
\text { (PP) }\end{array}$ & $\begin{array}{l}\text { University } \\
\text { Administrators } \\
\text { (UA) }\end{array}$ & $\begin{array}{l}\text { Hospital } \\
\text { Administrators } \\
\text { (HA) }\end{array}$ & Total \\
\hline Culture & $\begin{array}{l}103 \\
(21 \%)\end{array}$ & $\begin{array}{l}61 \\
(33 \%)\end{array}$ & $\begin{array}{l}73 \\
(30 \%)\end{array}$ & $\begin{array}{l}64 \\
(27 \%)\end{array}$ & \\
\hline $\begin{array}{l}\text { Physician } \\
\text { Workflow }\end{array}$ & $\begin{array}{l}117 \\
(24 \%)\end{array}$ & $\begin{array}{l}58 \\
(31 \%)\end{array}$ & $\begin{array}{l}35 \\
(15 \%)\end{array}$ & $\begin{array}{l}43 \\
(18 \%)\end{array}$ & \\
\hline $\begin{array}{l}\text { Outcomes and } \\
\text { Research }\end{array}$ & $\begin{array}{l}69 \\
(14 \%)\end{array}$ & $\begin{array}{l}6 \\
(3 \%)\end{array}$ & $\begin{array}{l}41 \\
(17 \%)\end{array}$ & $\begin{array}{l}57 \\
(24 \%)\end{array}$ & \\
\hline Patient Care & $\begin{array}{l}59 \\
(12 \%)\end{array}$ & $\begin{array}{l}18 \\
(10 \%)\end{array}$ & $\begin{array}{l}35 \\
(15 \%)\end{array}$ & $\begin{array}{l}41 \\
(17 \%)\end{array}$ & \\
\hline Communication & $\begin{array}{l}66 \\
(13 \%)\end{array}$ & $\begin{array}{l}27 \\
(14 \%)\end{array}$ & $\begin{array}{l}27 \\
(11 \%)\end{array}$ & $\begin{array}{l}21 \\
(9 \%)\end{array}$ & \\
\hline Education/Learning & $\begin{array}{l}77 \\
(16 \%)\end{array}$ & $\begin{array}{l}15 \\
(8 \%)\end{array}$ & $\begin{array}{l}30 \\
(12 \%)\end{array}$ & $\begin{array}{l}15 \\
(6 \%)\end{array}$ & \\
\hline Total & 491 & 185 & 241 & 241 & 1158 \\
\hline
\end{tabular}




\section{References}

1. Kohn Lea. To err is human : National Academy Press; 1999.

2. Institute of Medicine Committee on Quality of Health Care in America. Crossing the quality chasm: A new health system for the 21st Century. 1st ed.: National Academies Press; 2001.

3. Safety, Committee on Data Standards for Patient. Patient safety: achieving a new standard for care. 1st ed.: National Academies Press; 2004.

4. HIMSS Definitions [Internet]. Chicago: Healthcare Information and Management Systems; [cited 2011 Mar 23]. Available from http://www.himss.org/ASP/topics_ehr.asp.

5. Bates DW, Ebell M, Gotlieb E, Zapp J, Mullins HC. A proposal for electronic medical records in U.S. primary care. J Am Med Inform Assoc 2003; 10(1): 1-10.

6. Jha AK, Doolan D, Grandt D, Scott T, Bates DW. The use of health information technology in seven nations. Int J Med Inform 2008; 77(12): 848-854.

7. Executive Order 13410: Promoting Quality and Efficient Health Care in Federal Government Administered or Sponsored Health Care Programs [Internet]. Washington: Federal Register; [updated 2006 Aug 26; cited 2011 Mar 23]. Available from http://edocket.access.gpo.gov/2006/pdf/06-7220.pdf.

8. State of the Union [Internet]. Washington: The White House; [updated 2010 Jan 27; cited 2011 Mar 23]. Available from: http://www.whitehouse.gov/the-press-office/remarks-president-state-union-address.

9. Blumenthal D. Stimulating the adoption of health information technology. N Engl J Med 2009; 360(15): 1477-1479.

10. Ford EW, Menachemi N, Peterson LT, Huerta TR. Resistance is futile: but it is slowing the pace of EHR adoption nonetheless. J Am Med Inform Assoc 2009; 16(3): 274-281.

11. Lorenzi NM, Kouroubali A, Detmer DE, Bloomrosen M. How to successfully select and implement electronic health records (EHR) in small ambulatory practice settings. BMC Med Inform Decis Mak 2009; 9: 15 .

12. Rogers EM. Diffusion of innovations New York: Free Press; 1995.

13. Moore GA. Crossing the chasm : marketing and selling disruptive products to mainstream customers. Rev. ed. New York, NY: HarperBusiness Essentials; 2002.

14. Lorenzi NM, Novak LL, Weiss JB, Gadd CS, Unertl KM. Crossing the implementation chasm: a proposal for bold action. J Am Med Inform Assoc 2008; 15(3): 290-296.

15. Lorenzi NM. Strategies for creating successful local health information infrastructure Initiatives. 2003.

16. Lorenzi NM, Riley RT. Managing change: an overview. J Am Med Inform Assoc 2000; 7(2): 116-124.

17. Lorenzi NM, Riley RT. Organizational issues = change. Int J Med Inform 2003; 69: 197-203.

18. Hersh. Health care information technology: progress and barriers. J Am Med Inform Assoc 2004; 292: 2273.

19. Poon EG, Blumenthal D, Jaggi T, Honour MM, Bates DW, Kaushal R. Overcoming barriers to adopting and implementing computerized physician order entry systems in U.S. hospitals. Health Aff (Millwood) 2004; 23(4): 184-190.

20. Coye MJ, Kell J. How hospitals confront new technology. Health Aff (Millwood) 2006; 25: 163-173.

21. Hillestad R, Bigelow J, Bower A, Girosi F, Meili R, Scoville R, et al. Can electronic medical record systems transform health care? Potential health benefits, savings, and costs. Health Aff (Millwood) 2005; 24(5): $1103-1117$.

22. Glaser BG, Strauss AL editors. The discovery of grounded theory : strategies for qualitative research. Chicago: Aldine Pub. Co.; 1967.

23. Corbin J, Strauss A. Basics of Qualitative Research: Techniques and Procedures for Developing Grounded Theory. 3rd ed.: Sage Publications, Inc; 2007.

24. Krueger PRA, Casey DMA. Focus Groups: A Practical Guide for Applied Research 3rd ed.: Sage Publications, Inc; 2000.

25. Ash JS, Berg M, Coiera E. Some unintended consequences of information technology in health care: the nature of patient care information system-related errors. J Am Med Inform Assoc 2004; 11: 104-112.

26. Ash JS, Sittig DF, Poon EG, Guappone K, Campbell E, Dykstra RH. The extent and importance of unintended consequences related to computerized provider order entry. J Am Med Inform Assoc 2007; 14: 415-423.

27. Ash JS, Bates DW. Factors and forces affecting EHR system adoption: report of a 2004 ACMI discussion. J Am Med Inform Assoc 2005; 12: 8-12.

28. Ash JS, Sittig DF, Campbell E, Guappone K, Dykstra RH. An unintended consequence of CPOE implementation: Shifts in power, control, and autonomy. AMIA Annu Symp Proc 2006: 11-15.

29. Ash JS, Sittig DF, Dykstra RH, Guappone K, Carpenter JD, Seshadri V. Categorizing the unintended sociotechnical consequences of computerized provider order entry. Int J Med Inform 2007; 76 (Suppl. 1):21-27. 
30. Campbell EM, Sittig DF, Ash JS, Guappone KP, Dykstra RH. Types of unintended consequences related to computerized provider order entry. J Am Med Inform Assoc 2006; 13: 547-556.

31. Sittig DF, Krall M, Kaalaas-Sittig J, Ash JS. Emotional aspects of computer-based provider order entry: a qualitative study. J Am Med Inform Assoc 2005; 12: 561-567.

32. Joos D, Chen Q, Jirjis J, Johnson KB. An electronic medical record in primary care: impact on satisfaction, work efficiency and clinic processes. AMIA Annu Symp Proc 2006: 394-398.

33. O'Connell RT, Cho C, Shah N, Brown K, Shiffman RN. Take note(s): differential EHR satisfaction with two implementations under one roof. J Am Med Inform Assoc 2004; 11: 43-49.

34. Middleton B, Hammond WE, Brennan PF, Cooper GF. Accelerating U.S. EHR adoption: how to get there from here. recommendations based on the 2004 ACMI retreat. J Am Med Inform Assoc 2005; 12: 13-19.

35. Middleton B. Achieving US health information technology adoption: The need for a third hand. Health Affairs 2005; 24: 1269.

36. van der Meijden MJ, Tange H, Troost J, Hasman A. Development and implementation of an EPR: how to encourage the user. Int J Med Inform 2001; 64: 173-185.

37. Ford EW, Menachemi N, Phillips MT. Predicting the adoption of electronic health records by physicians: when will health care be paperless? J Am Med Inform Assoc 2006; 13: 106-112.

38. Report to the president realizing the full potential of health information technology to improve healthcare for americans : The path forward [internet]. Washington: President's Council of Advisors on Science and Technology; [updated 2010 Dec 8; cited 2011 Mar 23]. Available from: http://www.whitehouse.gov/sites/de fault/files/microsites/ostp/pcast-health-it-report.pdf 